\title{
EFFECTIVE USE OF NEXT GENERATION SEQUENCING FOR GENETIC DIAG- NOSIS OF A PATIENT WITH A MOSAIC TSC2 VARIANT
}

\author{
Hilal Sena Çifcibaş1 ${ }^{1}$, Elçin Gülbal ${ }^{1}$, Ayşenur Erdoğan ${ }^{1}$, Ayşenur Demirci ${ }^{1}$, Selma Demir ${ }^{2}$
}

${ }^{1}$ Trakya University School of Medicine, Edirne, TURKEY

${ }^{2}$ Department of Medical Genetics, Trakya University School of Medicine, Edirne, TURKEY

\section{ABSTRACT}

Aims: Tuberous sclerosis complex is a genetic disorder characterized by mutations in Tuberous Sclerosis Complex $1\left({ }^{*} 605284\right)$ or Tuberous Sclerosis Complex $2\left({ }^{*} 191092\right)$ genes. PALB2 $\left({ }^{*} 610355\right)$ gene has long been known to be associated with increased breast and pancreatic cancer risk. Its association with risk of stomach and lung cancers has also been established recently. In this case report, it is aimed to present a case of a 24 -year-old female patient, who has been diagnosed with both novel mosaic Tuberous Sclerosis Complex 2 pathogenic variation and pathogenic PALB2 variation at the same time.

Case Report: A 24-year-old female patient was admitted to Trakya University Genetic Diseases Center with suspected tuberous sclerosis complex. Her primary complaints were a continuous headache and fatigue. She had no history of convulsion and seizure. There were bilateral facial angiofibromas around her nose. When genomic DNA was isolated, both novel mosaic Tuberous Sclerosis Complex 2 pathogenic variation and pathogenic PALB2 variation were observed.

Conclusion: This case report shows that in cancer predisposing syndromes, Next-generation sequencing is a powerful technique to portrait a detailed genetic profile of patient, investigating for any mosaicism or other risk alleles.

Keywords: Tuberous sclerosis, mosaicism, mutation

\section{INTRODUCTION}

Tuberous sclerosis complex (TSC) is a genetic disorder characterized by mutations in TSC1 $\left({ }^{\star} 605284\right)$ or TSC2 $\left.*^{*} 191092\right)$ genes (1). TSC1 gene $\left({ }^{*} 605284\right)$ encodes hamartin protein while TSC2 gene (OMIM) encodes tuberin protein $(1,2)$. Heterozygous mutations in these two genes are found in about 75\%-90\% (31\% of mutations are found in TSC1 gene and 69\% of mutations are in TSC2 gene) according to the TSC clinical diagnostic criteria (1). The overall incidence of TSC is $1 / 6,000$ to $1 / 10,000$ live births (2).

Diagnosis of TSC is mainly based on clinical findings, including the formation of hamartomas in different organs. Tubers and nodules in brain and retina, facial angiofibromas, hypomelanotic macules and fibromas of the skin, cardiac rhabdomyomas, renal angiomyoli- pomas, and pulmonary lymphangioleiomyomatosis are also other common clinical manifestations of TSC (2).

Located in 16p12, PALB2 $\left({ }^{\star} 600185\right)$ gene, whose product colocalizes with BRCA2 gene in nuclear foci, has recombinational repair and checkpoint functions. The association between PALB2 gene and risk of breast and pancreatic cancer has long been recognized. Moreover, PALB2 gene has recently been suggested to be associated with increased risk of stomach and lung cancers $(3,4)$.

Next-generation sequencing (NGS) is a practical and cost-effective method for screening mutations in large genes and many genes at the same time. The other advantage of this technique is its ability to detect mosaic variants. Next-generation panels contain different genes that can be analyzed in a single reaction. These genes can be responsible for overlapping clinical mani- 
festations, like neurofibromatosis and TSC (5).

In this case report, a 24-year-old female patient is presented who has been diagnosed with having both novel mosaic TSC2 pathogenic variation and pathogenic PALB2 variation at the same time.

\section{CASE REPORT}

A 24-year-old female patient was admitted to Trakya University Genetic Diseases Center with TSC as a differential diagnosis. The patient was the only child of a non-consanguineous Turkish couple. Patient's primary complaints were a continuous headache and fatigue. The patient had no history of convulsion and seizure. There were bilateral facial angiofibromas around her nose (Figure 1). Hyperintense foci were found in her left temporal region in T2A and fluid-attenuated inversion-recovery sequences of magnetic resonance imaging scan, which are compatible with cortical tubers. The patient also had angiomyolipoma on her right kidney.

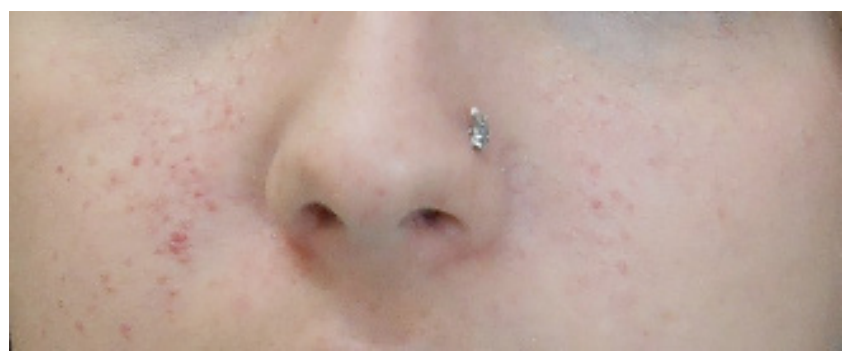

Figure 1: Bilateral facial angiofibromas on the nasolabial folds.

After obtaining written informed consent, genomic DNA was isolated using EZ1 automated DNA isolation system (Qiagen) from peripheral blood samples of the patient and patient's family members. DNA Quantification was performed with Qubit ${ }^{\oplus}$ dsDNA HS Assay Kit (Invitrogen) according to the instructions of the manufacturer. TruSight Rapid Capture Kit (Illumina) was used for target enrichment of 94 genes (Trusight Cancer Kit, Illumina) including both TSC1 and TSC2. $150 \mathrm{bp}$ paired-end reads were sequenced and aligned on Miseq System. Variant calling and analysis was performed on Genomize-Seq (Turkey) platform. Because of the probability that TSC1 and TSC2 variants might be mosaic in the patient, fastq files were analyzed on base space with Cancer Variant Caller (Illumina) and visualized data on IGV (Broad Institute). Mutation confirmation and segregation analysis was made by Sanger Sequencing on ABI 3130 XL. Variants have been analyzed in silico by using Varsome and classified according to the ACMG 2015 criteria (6).

A mosaic TSC2 in-frame deletion encompassing $9 \mathrm{bp}$ around the splice site of exon 34 was found (NM_000548.4(TSC2):c.4006-2_4012delAGTCGTC$\mathrm{CT}$ ) (Figure 2A). The frequency of the mutated allele was about $10 \%$ (38/300 coverage). In addition, the patient was heterozygous for NM_024675.3(PALB2):c.1704_1707delAAAA (p.Lys569Argfs) variation (Figure $2 \mathrm{~B}$ ) which has been reported as "pathogenic" in ClinVar database previously.

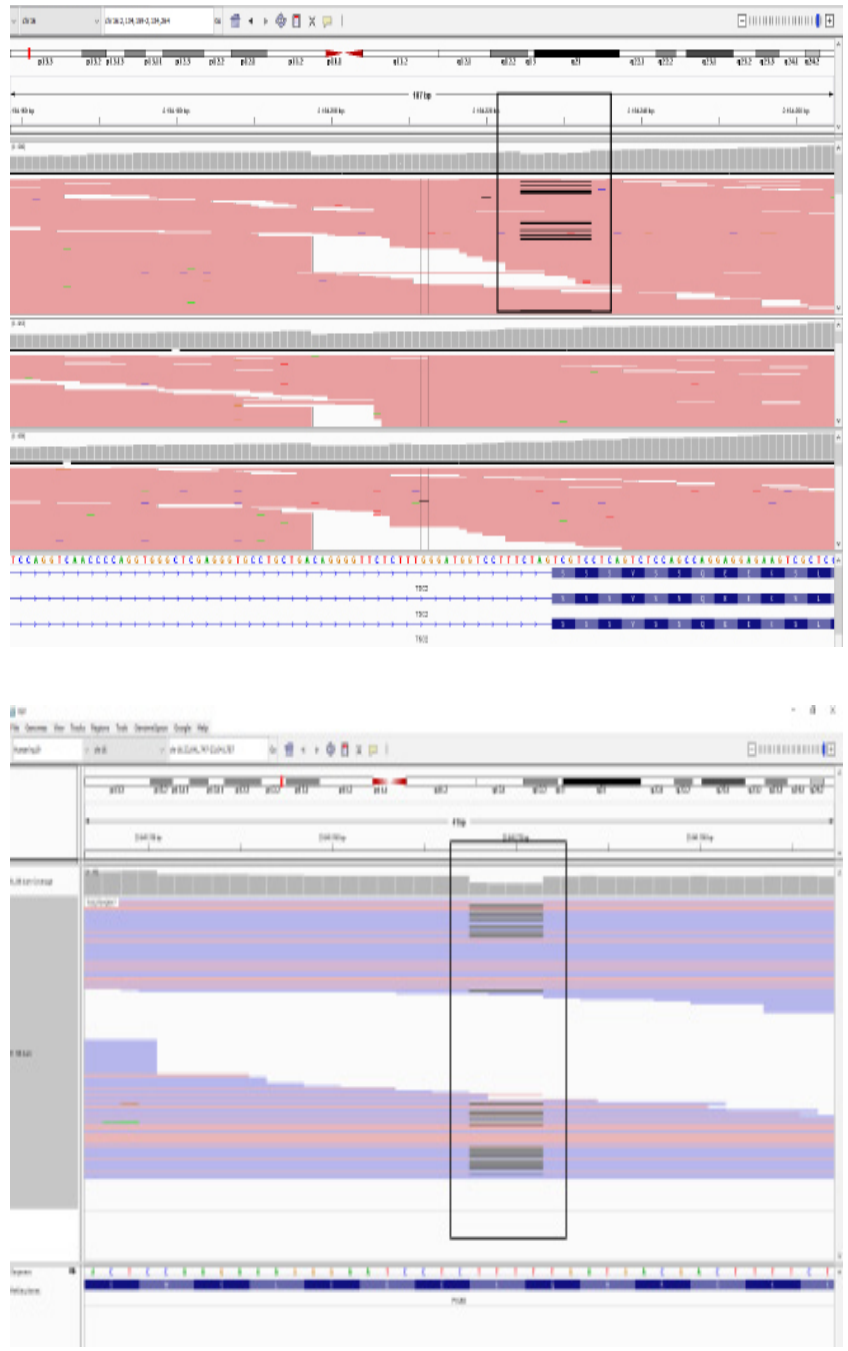

Figure 2: A mosaic TSC2 in-frame deletion encompassing $9 b p$ around the splice site of exon 34(NM_000548.4(TSC2):c.4006-2_4012delAGTCGTCCT) (A), Heterozygous NM_024675.3(PALB2):c.1704_1707delAAAA (p.Lys569Argfs) variation (B). 
On the contrary, based on results from sequencing, a mutation in TSC2 gene was not present in genome of the patient's parents. However, the patient had some small raw peaks near the base of the sequencing baseline and these raw peaks were compatible with the deleted allele of the patient. On the other hand, the patient's mother was a carrier of PALB2 pathogenic variation, whereas her father did not have any mutation in this region (Figure 3). Her mother does not have any cancer history or familial history of cancer. Close clinical follow-up was offered to both the patient and her mother, in particular assessment of any features of cancer.

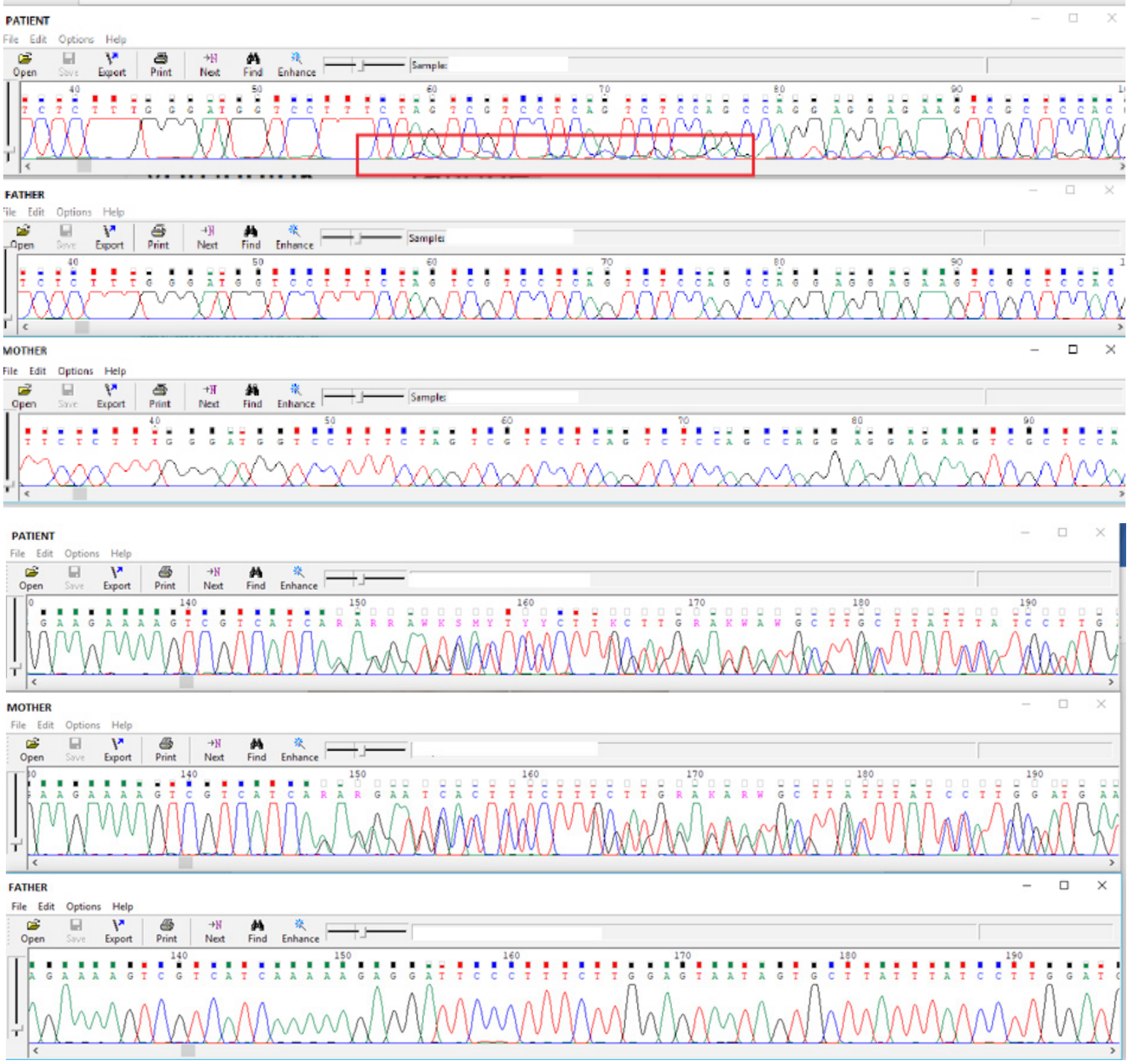

Figure 3: Small raw peaks near the base of the sequencing baseline and these raw peaks (red framed region) were compatible with the deleted allele and normal electropherogram of parents, mother was carrying the PALB2 pathogenic variation, whereas the father is normal for this region. 


\section{DISCUSSION}

A number of mutations in TSC1 and TSC 2 genes have been reported in the literature, including somatic pathogenic variants in patients who are clinically diagnosed with TSC (1). The patient reported in the present case report was diagnosed with mosaic TSC2 in pathogenic variant. She was also heterozygous for a previously described pathogenic PALB2 gene variation. To the best of our knowledge, this is the first case report in the literature that identified a patient with concurrent mosaic pathogenic TSC2 variant and pathogenic PALB2 gene variant at the same time.

Mosaic variants in TSC1 and TSC2 genes are known to be associated with a milder phenotype of TSC. Clinical manifestations of TSC differ among patients, depending on the degree of the mosaic variant. Milder clinical phenotype of our patient may be attributed to the low level of mutated allele ratio. It is difficult to determine low frequency mosaic mutation by conventional methods like Sanger sequencing. In comparison, NGS is an easier and faster method to detect mosaic variations (1). The precise determination of mosaic variant in our patient with NGS supports the effectiveness of this technology for the diagnosis of clinically suspected TSC.

Incidental findings should be reported to the patients in case of identifying any pathogenic variant at an important locus (7). The finding of PALB2 mutation by NGS when searching for TSC in our patient is described as an incidental finding. Because PALB2 is an important cancer predisposing gene, the patient and the patient's mother are given a detailed genetic counseling and referred to close clinical follow-up for assessing and monitoring risk of cancer.

In conclusion, we suggest that in cancer predisposing syndromes, NGS is a powerful technique to portrait a detailed genetic profile of patient, investigating for any mosaicism or other risk alleles.

\section{Ethics Committee Approval: N/A}

Informed Consent: Written informed consent was obtained from the patient of this study.

Conflict of Interest: The authors declared no conflict of interest.

Author contributions: Concept: HSÇ, EG, AE, AD, SD. Design: HSÇ, EG, AE, AD, SD. Supervision: HSÇ, EG, AE, AD, SD. Resources: HSÇ, EG, AE, AD, SD. Materi- als: HSÇ, EG, AE, AD, SD. Data collection and/ or processing: HSÇ, EG, AE, AD, SD. Analysis and/or Interpretation: HSÇ, EG, AE, AD, SD. Literature Search: HSÇ, EG, AE, AD, SD. Writing Manuscript: HSÇ, EG, AE, AD, SD. Critical Review: HSÇ, EG, AE, AD, SD.

Financial disclosure: The authors declared that this study received no financial support.

Editor-in-chief's Note: One of the author of this article, Hilal Sena Çifcibaşı is a member of the editorial board of Turkish Medical Student Journal. However, she did not take place in any stage on the editorial decision of the manuscript. The editors who evaluated this manuscript are from another institutions.

\section{REFERENCES}

1. Nellist M, Brouwer RW, Kockx CE et al. Targeted Next generation sequencing reveals previously unidentified TSC1 and TSC2 mutations. BMC Med Genet 2015;16:10.

2. Avgeris S, Fostira F, Vagena A et al. Mutational analysis of TSC1 and TSC2 genes in tuberous sclerosis complex patients from Greece. Sci Rep 2017;7(1):16697.

3. Lee JEA, Li N, Rowley SM et al. Molecular analysis of PALB2-associated breast cancers. J Pathol 2018;245(1):53-60.

4. Bleuyard JY, Butler RM, Esashi F. Perturbation of PALB2 function by the T413S mutation found in small cell lung cancer. Wellcome Open Res 2017;2:110.

5. Serratì S, De Summa S, Pilato B et al. Next-generation sequencing: advances and applications in cancer diagnosis. Onco Targets and Ther 2016;9:7355-65.

6. Richards S, Aziz N, Bale S et al. Standards and guidelines for the interpretation of sequence variants: a joint consensus recommendation of the American College of Medical Genetics and Genomics and the Association for Molecular Pathology. Genet Med 2015;17(5):40524.

7. Blackburn HL, Schroeder B, Turner C et al. Management of incidental findings in the era of Next-generation sequencing. Curr Genomics 2015;16(3):159-74. 